\title{
Effects of training frequency on muscular strength for trained men under volume matched conditions
}

\author{
Emil Johnsen ${ }^{1}$, Roland van den Tillaar ${ }^{\text {Corresp. } 1}$ \\ ${ }^{1}$ Department of Sport Sciences and Physical Education, Nord University, Levanger, Norway \\ Corresponding Author: Roland van den Tillaar \\ Email address: roland.v.tillaar@nord.no
}

In resistance training, the role of training frequency to increase maximal strength is often debated. However, the limited data available does not allow for clear training frequency "optimization" recommendations. The purpose of this study was to investigate the effects of training frequency on maximal muscular strength and rate of perceived exertion (RPE). The total weekly training volume was equally distributed between two or four sessions per muscle group. Methods Twenty-one experienced resistance-trained male subjects (height: $1.85 \pm 0.06 \mathrm{~m}$, body mass: $85.3 \pm 12.3 \mathrm{~kg}$, age: $27.6 \pm 7.6$ years) were tested prior to and after an eight-week training period in one-repetition maximum (1RM) barbell back squat and bench press. Subjects were randomly assigned to a SPLIT group ( $n=10)$, in which there were two training sessions of squats and lower-body exercises and two training sessions of bench press and upper-body exercises, or a FULLBODY group ( $n=11$ ), in which four sessions with squats, bench press and supplementary exercises were conducted every session. In each session, the subjects rated their RPE after barbell back squat, bench press, and the full session. Results Both groups significantly increased 1RM strength in barbell back squat (SPLIT group: $+13.25 \mathrm{~kg}$; FULLBODY group: $+14.31 \mathrm{~kg}$ ) and bench press (SPLIT group: $+7.75 \mathrm{~kg}$; FULLBODY group: $+8.86 \mathrm{~kg}$,) but training frequency did not affect this increase for squat $(p=0.640)$ or bench press $(p=0.431)$. Both groups showed a significant effect for time on RPE on all three measurements. The analyses showed only an interaction effect between groups on time for the RPE after the squat exercise $(p=0.002)$. Conclusion We conclude that there are no additional benefits of increasing the training frequency from two to four sessions under volume-equated conditions, but it could be favorable to spread the total training volume into several training bouts through the week to avoid potential increases in RPE, especially after the squat exercise. 
1 Effects of training frequency on muscular strength for trained men under

2 volume matched conditions

3

4 Conflict of interest disclosure: None.

5

6 Original research

7

8

9 Authors: Emil Johnsen, Roland van den Tillaar ${ }^{凶}$

10 Department of Sport Sciences and Physical Education, Nord University, Levanger, Norway ${ }^{凶}$

11

$12 \square$ Corresponding author

13 Prof. Roland van den Tillaar PhD.

14 Department of Sports Sciences and Physical Education

15 Nord University

16 Odins veg 23

$17 \quad 7603$ Levanger

18 Norway

19 E-mail: roland.v.tillaar@nord.no

20 Phone: $+47-97662913$

21 Fax: 0047-7411 2001 


\section{ABSTRACT}

\section{Background}

25 In resistance training, the role of training frequency to increase maximal strength is often debated. 26 However, the limited data available does not allow for clear training frequency "optimization" 27 recommendations. The purpose of this study was to investigate the effects of training frequency 28 on maximal muscular strength and rate of perceived exertion (RPE). The total weekly training 29 volume was equally distributed between two or four sessions per muscle group.

30 Methods

31 Twenty-one experienced resistance-trained male subjects (height: $1.85 \pm 0.06 \mathrm{~m}$, body mass: 85.3 $32 \pm 12.3 \mathrm{~kg}$, age: $27.6 \pm 7.6$ years) were tested prior to and after an eight-week training period in 33 one-repetition maximum (1RM) barbell back squat and bench press. Subjects were randomly 34 assigned to a SPLIT group $(\mathrm{n}=10)$, in which there were two training sessions of squats and lower35 body exercises and two training sessions of bench press and upper-body exercises, or a 36 FULLBODY group $(\mathrm{n}=11)$, in which four sessions with squats, bench press and supplementary 37 exercises were conducted every session. In each session, the subjects rated their RPE after barbell 38 back squat, bench press, and the full session.

\section{Results}

40 Both groups significantly increased 1RM strength in barbell back squat (SPLIT group: $+13.25 \mathrm{~kg}$; 41 FULLBODY group: +14.31 kg) and bench press (SPLIT group: $+7.75 \mathrm{~kg}$; FULLBODY group: $42+8.86 \mathrm{~kg}$, ) but training frequency did not affect this increase for squat $(\mathrm{p}=0.640)$ or bench press $43(\mathrm{p}=0.431)$. Both groups showed a significant effect for time on RPE on all three measurements. 44 The analyses showed only an interaction effect between groups on time for the RPE after the squat 45 exercise $(\mathrm{p}=0.002)$.

\section{Conclusion}

47 We conclude that there are no additional benefits of increasing the training frequency from two to 48 four sessions under volume-equated conditions, but it could be favorable to spread the total training 49 volume into several training bouts through the week to avoid potential increases in RPE, especially 50 after the squat exercise. 
52 KEY WORDS: RPE, rate of perceived exertion, bench press, squats 


\section{INTRODUCTION}

55 The interest in resistance training has risen in popularity (Wernbom et al., 2007). Several studies

56 pointed out that conducting resistance training had many potential health benefits for people of all

57 ages (Winett \& Carpinelli, 2001). An increase in overall strength through resistance training is

58 also seen in the context of an increase among athletes in a variety of sports (Suchomel et al., 2016).

59 Resistance training is an important factor in maintaining and developing muscle mass and muscle

60 strength. To maximize these adaptations in human muscles, the manipulations of various resistance

61 training variables (e.g. volume, intensity, load, and frequency) are key (Kraemer \& Ratamess,

62 2004). Manipulations to training intensity and volume have received most of the attention but

63 training frequency has largely been overlooked (Grgic et al., 2018; Ralston et al., 2017).

64 The role of training frequency has been debated, and the optimal frequency is not clear. Training

65 frequency is defined in the literature as the number of training sessions performed for a given

66 period, usually described on a weekly basis (Kraemer \& Ratamess, 2004). Frequency has be further

67 characterized by the number of training sessions per week per muscle group or exercise

68 (Schoenfeld et al., 2015), which is the definition used in this article. The American College of

69 Sports Medicine (2009), recommends that novices and untrained individuals should train every

70 muscle group 2-3 times per week (1). However, this recommendation of training frequency has

71 been the subject of some criticism since it is based on limited evidence (Grgic et al., 2018; Ralston

72 et al., 2018; Schoenfeld et al., 2019b). As a result, there has been a small renaissance on training

73 frequency, with multiple studies published on the topic. One study that has received much attention

74 was the "Norwegian Frequency Project," which showed positive results favoring higher frequency

75 training for elite/trained powerlifters (Raastad, 2012). The problem with this study is that it was

76 only used as a conference paper and never published in a journal, so it is difficult to control and

77 verify the methods used in the project. 
78 Although the number of studies published is increasing, the total pool of studies is still limited. To

79 the best of our knowledge, there are eight published studies that explore the effects of training

80 frequencies on muscle adaptations on trained males under equal volume conditions (Brigatto et al.,

81 2019; Colquhoun et al., 2018; Gentil et al., 2018; Gomes et al., 2019; Lasevicius et al., 2019;

82 Mclester et al., 2000; Saric et al., 2019; Schoenfeld et al., 2015). Several of these studies have

83 focused on lower training frequencies, i.e. three or lower. Only three of these studies controlled

84 for the effect of training frequencies higher than three (Colquhoun et al., 2018; Gomes et al., 2019;

85 Saric et al., 2019). Furthermore, most of these studies did not find any differences in gains in 1RM

86 between training frequencies except Mclester et al. (2000), who reported that the gains in 1RM by

87 training once a week were $33 \%$ lower than training three times per week. However, in this study

88 the volume was very low compared with the other studies and men and women were combined,

89 which could influence the results.

90 Two recent meta-analyses noted that the literature on training frequency under equal volume

91 conditions is small and suggested that future research is needed (Grgic et al., 2018; Ralston et al.,

92 2018). Ralston et al. (2018) noted that studies with trained subjects were needed. Furthermore,

93 Dankel et al. (2017) suggested that an increase in training frequency could be advantageous to

94 spread the total training volume to counteract muscle fatigue and overtraining. Training with a

95 very high volume in one training session can induce high levels of fatigue and prolonged recovery

96 time, which can be suboptimal for athletes that try to induce specific neuromuscular adaptations

97 (Pareja-Blanco et al., 2018). Seen in the context of motor learning theory, it also can be assumed

98 that more frequent training of a movement could lead to a higher increase in strength, due to an

99 improvement in neural efficiency (Shea et al., 2000). 
100 Since it is unclear whether exercise frequency affects muscular strength under equal total exercise

101 volume, especially in higher training frequencies (Grgic et al., 2018), the purpose of this paper was

102 two-fold: first, to investigate the effect of training frequency of two versus four times per week

103 when matched on total training volume upon maximal muscle strength in strength-trained males;

104 second, to investigate the effects of training frequency on perceptual responses (rate of perceived

105 exertion) among the subjects. We hypothesized that training with a frequency of four sessions per

106 week would promote greater increases in maximal strength with a lower self-reported rate of

107 perceived exertion (RPE) compared to two sessions per week due to the lower workload per muscle

108 group per training session.

109

\section{METHODS}

\section{Subjects}

112 Subjects were 21 male volunteers (height: $1.85 \pm 0.06 \mathrm{~m}$, body mass: $85.3 \pm 12.3 \mathrm{~kg}$, age: $27.6 \pm$

1137.6 years) who were recruited subjects that attend the local gym. The inclusion criteria were the

114 subject had to be male, could be defined as trained (a least one year experience of resistance

115 training with a minimum of two workouts per week) with experience training on barbell back squat

116 and bench press, was free of injuries, and stated they had not taken any performance enhancing

117 drugs. The mean resistance training age of the group was $4.7 \pm 2.8$ years. Each subject was

118 informed of the testing protocol, training procedures, and possible risks; and written consent was

119 obtained from the subjects prior to the study. The study was conducted with the approval of the

120 Norwegian Center for Research Data project number: 42440 and conformed to the latest revision

121 of the Declaration of Helsinki. 


\section{Study design}

124 To investigate the effect of training frequency with the same training volume upon strength (1

125 repetition maximum in the bench press and squats) and RPE, a pretest-posttest randomized group

126 design was used. Subjects were randomly assigned to one of two experimental groups: a SPLIT

127 group where the training protocol was divided into two sessions training barbell back squat and

128 exercises for the lower body and two sessions training bench press and exercises for the upper

129 body; or a FULLBODY group where subjects trained four full-body sessions with barbell back

130 squat and bench press each time, together with four other supplementary exercises for the whole

131 body. A summary of the resistance training protocol can be found in Table 1. Throughout the eight-

132 week training period, all resistance training variables were held constant, especially total training

133 volume (repetitions x set $\mathrm{x}$ intensity), between the two conditions, except the training frequency.

134 The training protocol was built up with a pretest the week before the training period and a posttest

135 the week after.

\section{Procedures}

138 One week before and after the intervention period, maximal strength was assessed by a one-

139 repetition maximum $(1 \mathrm{RM})$ test in barbell back squat $\left(1 \mathrm{RM}_{\mathrm{SQUAT}}\right)$ and bench press

140 (1RM $\left.\mathrm{BENCHPRESS}_{\mathrm{B}}\right)$. The 1RM test was done following the guidelines established by the National

141 Strength and Conditioning Association (Haff et al., 2016). The subjects started with a 5-10-minute

142 general warm-up consisting of running on a treadmill, followed by a set of five repetitions at

143 around $50 \%$ of an estimated $1 \mathrm{RM}$ and $2-3$ sets of $2-3$ repetitions around $60-80 \%$ of the estimated

144 1RM. The subjects then performed one repetition sets with increasing load to establish their 1RM. 
145 They had a maximum of five attempts to determine the 1RM. 1RM in barbell back squat was

146 always tested first followed by testing 1RM in bench press. Between each successful attempt, the

147 subject rested for 3-5 minutes before the next set with increased weight. To get an attempt

148 approved in the barbell back squat, the subjects had to meet the parallel depth and a green light

149 from the test leader. In bench press, the subject had to have head, shoulders, and bottoms placed

150 on the bench and the feet placed on the floor during the lift. They had to lower the barbell to their

151 chest and had to achieve full extension in the elbow to get the lift approved. The subjects were

152 asked to refrain from any other exercise for 24 hours before testing.

153 A rating of perceived exertion (RPE) was used to test if there was a difference between the two

154 groups after the exercises and workouts. RPE scales have been well-established as methods of

155 determining exertion during exercise (Helms et al., 2016). The Borg CR10 scale was used as the

156 RPE scale to quantify the perception of physical exertion (Morishita et al., 2013). After completing

157 each of the exercises (barbell back squat and bench press), and a couple of minutes after the

158 exercise session, the subject was instructed to rate their perceived exertion by choosing a number

159 on the CR10 scale. A rating of 0 was categorized as no exertion or at rest; a rating of 10 was the

160 maximal exertion they can achieve. The RPE measurement for each of the three rating points was

161 analyzed as a weekly mean for each of the subjects. All ratings through the training week for each

162 of the three measurements were summed and divided by the total number of measurements in that

163 week. The subjects were familiar with using the CR10 scale since they regularly evaluated their

164 training intensity by using this scale.

165 After the pretest, the subjects were randomly assigned to one of the two experimental groups:

166 SPLIT ( $\mathrm{n}=10$, height: $1.84 \pm 0.05 \mathrm{~m}$, body mass: $87.0 \pm 13.3 \mathrm{~kg}$, age: $30.6 \pm 9.5 \mathrm{yrs}$.) and

167 FULLBODY $(\mathrm{n}=11$, height: $1.87 \pm 0.07 \mathrm{~m}$, body mass: $83.7 \pm 11.6 \mathrm{~kg}$, age: $24.8 \pm 4.0$ yrs.); 
168 each group trained under matched volumes. To control for volume, the total weekly resistance

169 training volume (repetitions $\mathrm{x}$ set $\mathrm{x}$ intensity) was equated between the groups. The volume was

170 equated because a dose-response relationship between volume and increase in muscular strength

171 has been previously reported (Heaselgrave et al., 2019; Ralston et al., 2018; Rhea et al., 2003).

172 The SPLIT training group trained with a frequency of two sessions per muscle group; the training

173 protocol was divided into two lower-body and two upper-body workouts. The FULLBODY

174 training group had a training frequency of four sessions per muscle group; they trained four full-

175 body workouts per week.

176 The training protocol for both groups also included a mixture of single- and multi-joint exercises

177 for the rest of the body (Table 1). The weekly total training volume of the two groups was equal.

178 Back squat and bench press had the training intensity determined based on their one-repetition

179 maximum (1RM) and was a percentage of the $1 \mathrm{RM}$. The repetition ranges for these exercises were

180 between two and ten, with the higher repetitions in the first of weeks. The intensity was between

$18165 \%$ and $90 \%$ of their $1 \mathrm{RM}$. The intensity and repetition ranges for the bench press and back squat

182 was periodized with more repetition and lower intensity during the first weeks; throughout the

183 training period, the repetitions and intensity gradually shifted to fewer repetitions with high

184 intensity. Weekly repetition ranges and intensity is reported in Table 2. The weekly set volume of

185 these exercises was set to eleven, which follows the recommendation from Ralston et al. (2017),

186 who recommended a medium to high weekly set count for maximizing strength gain.

187 In the other exercises, the repetition ranges were between eight and twelve repetitions, with the 188 intensity set to two repetitions in reserve, i.e., is the number of repetitions the athlete feels he has

189 left in the tank. This means that with the two repetitions in reserve, the subject had to feel that he

190 had a maximum of two repetitions left to fatigue after a set of one exercise on that weight (Zourdos 
191 et al., 2016). If the subject felt that he could do more, he was instructed to increase the weight by

$192-10 \%$ on the next set. The two repetitions in reserve method for the supplementary exercises was

193 used to have the possibility for autoregulation within each subject and to avoid that the subject

194 performed until full exhaustion which could influence training the main exercises.

TABLE 1 AND 2 AROUND HERE

197

\section{Statistical Analyses}

199 All statistical analyses were performed using SPSS 25.0 for Windows (SPSS Inc., Chicago, IL, 200 USA). The normality and homogeneity of the variances were verified using the Shapiro-Wilk and

201 Levene's tests. Descriptive statistics (mean \pm SD) were calculated for each dependent variable for 202 the pretest and posttest $\left(1 \mathrm{RM}_{\mathrm{SQUAT}}, 1 \mathrm{R} \mathrm{M}_{\mathrm{BENCHPRESS}}\right)$. To compare the effect of the two protocols, 203 a two-way ANOVA of 2 (pretest and posttest) x 2 (groups: FULLBODY and SPLIT) was 204 performed for each of the strength assessments. The percentage increase was also calculated for $205 \quad 1 \mathrm{RM}_{\mathrm{SQUAT}}$ and $1 \mathrm{RM} \mathrm{BENCHPRESS}_{\mathrm{B}}$

206 A two-way ANOVA of 2 (groups: FULLBODY and SPLIT) x 8 (weekly mean of RPE, week 1 to 207 8) was performed for the three different RPEs (RPE after bench press, squat, and workout). 208 Assumptions of sphericity were evaluated using Mauchly's test; where sphericity was violated ( $p$ $209<0.05$ ), the Greenhouse-Geisser correction factor was applied. A one-way ANOVA (weekly mean 210 of RPE, week 1 to 8) was also done per group for the three different RPEs to identify the 211 development per group. When significant differences occurred, Holm-Bonferroni post hoc tests

212 were conducted to identify statistically significant comparisons. The level of significance was set 213 at $\mathrm{p}<0.05$, and all data were expressed as mean \pm SD. Effect size was evaluated with $\eta^{2}$ (eta 
214 squared) where $0.01<\eta^{2}<0.06$ constitutes a small effect, $0.06<\eta^{2}<0.14$ constitutes a medium

215 effect, and $\eta^{2}>0.14$ constitutes a large effect (Cohen, 1988).

216

\section{RESULTS}

218 At baseline, no significant difference in $1 \mathrm{RM}$ in squat $(\mathrm{p}=0.55)$ and bench press $(\mathrm{p}=0.46)$ were

219 found between the groups. Each group increased in the bench press (SPLIT group: $+7.75 \mathrm{~kg}$;

220 FULLBODY group: $+8.86 \mathrm{~kg} ; \mathrm{F}=223.9, \mathrm{p}<0.001, \eta^{2}=0.92$ ) and squat exercise (SPLIT group:

$221+13.25 \mathrm{~kg}$; FULLBODY group: $\left.+14.31 \mathrm{~kg} ; \mathrm{F}=152.9, \mathrm{p}<0.001, \eta^{2}=0.89\right)$ significantly from pre

222 to post test (Figure 1). Relatively, the SPLIT and FULLBODY training groups increased by $7.7 \%$

223 and $9.7 \%$, respectively, in $1 \mathrm{RM}$ bench press and by $12.1 \%$ and $11.5 \%$, respectively, in $1 \mathrm{RM}$ squats.

224 The relative gain in $1 \mathrm{RM}$ in squats was significantly higher than in $1 \mathrm{RM}$ in bench press $(\mathrm{p}=0.022)$.

225 The was no effect of group $\left(\mathrm{F} \leq 0.71, \mathrm{p} \geq 0.40, \eta^{2} \leq 0.036\right)$ or interaction effect of group*time (F $226 \leq 1.0, \mathrm{p} \geq 0.33, \eta^{2} \leq 0.05$ ) for strength (Figure 2).

227

228

FIGURE 1 AND 2 AROUND HERE

229

230 The rating of perceived exertion (RPE) for the exercise bout $\left(F=4.9, p<0.001, \eta^{2}=0.21\right)$, after

231 the squat $\left(F=10.2, p<0.001, \eta^{2}=0.35\right)$ and bench press $\left(F=3.0, p=0.043\right.$, partial $\left.n^{2}=0.14\right)$

232 exercises per week were influenced during the intervention period. Only a significant time* group 233 effect was found in RPE after the squat exercise $\left(F=3.5, p=0.002, \eta^{2}=0.15\right)$. In addition, a non-

234 significant but large effect was found between the two training groups in RPE after the squat 235 exercise $\left(F=3.34, p=0.083, \eta^{2}=0.15\right)$. 
236 The post hoc comparison revealed that the subjects in the split group reported significantly higher

237 session RPE in week 4 compared with week 3 and 5; the RPE increased again from week 6 to 8

238 (Figure 3). In the FULLBODY group, the session RPE decreased from week 1 to 2 followed by

239 an increase until week 4. In week 5, it decreased again significantly (Figure 3).

240 RPE after the squat was significantly higher in the SPLIT group in weeks 4 and 5 compared with

241 the FULLBODY group. Also, the development of the RPE per week after squats followed a

242 different development: while the RPE increased in week 4, decreased in week 5, and increased

243 again the last two weeks for the SPLIT group, the RPE of the FULLBODY group decreased to a

244 minimum in week 5, after which it increased again in week 6 (Figure 3).

245 RPE after bench press only changed in week two, in which only the FULLBODY group had a

246 significantly lower RPE compared with the other weeks during training. This also resulted in a 247 significant difference with the SPLIT group in week two (Figure 3).

FigURE 3 AROUND HERE

\section{DISCUSSION}

252 The main aim of this study was to investigate the effect of resistance training frequency on 253 maximal muscular strength and RPE by training twice versus four times a week when matched on 254 total training volume. The main findings were that both training frequencies achieved a similar 255 significant increase in maximal strength (1RM) in the barbell back squat and bench press over the 256 eight weeks of training. However, RPE developed differently during the training period in which, 257 especially after the squat exercise, RPE seems to be higher some weeks for the SPLIT group 258 compared with the FULLBODY group. 
259 Both SPLIT and FULLBODY groups had a similar increase in strength from pretest to posttest in

260 both $1 \mathrm{RM}_{\mathrm{SQUAT}}\left(13.25\right.$ and $12.27 \mathrm{~kg}$, respectively) and $1 \mathrm{RM}_{\mathrm{BENCHPRESS}}(7.75$ and $8.86 \mathrm{~kg}$,

261 respectively), which indicates that eight weeks of training, regardless of frequency, will increase

262 muscle strength, as long as the weekly training volume in the exercises, barbell back squat, and

263 bench press are high enough. The result of this study follows the trends shown in other studies

264 (Brigatto et al., 2019; Colquhoun et al., 2018; Gentil et al., 2018; Gomes et al., 2019; Lasevicius

265 et al., 2019; Saric et al., 2019; Schoenfeld et al., 2015) on the topic, with the effect of an increase

266 in frequency not yielding a significantly greater effect on maximal strength. Only Mclester et al.

267 (2000) reported that a lower frequency group achieved only $2 / 3$ of the increase in strength of the

268 high-frequency group, but they compared one session per week with three sessions per week.

269 Regardless of frequency, the relative 1RM gain in squats was higher than in the bench press. This

270 can be explained by two mechanisms. The first explanation could be in the difference in loading

271 schemes for the exercises. The protocol for bench press had a lower percentage of 1RM in the first

272 couple of weeks, which could have been a less optimal scheme than the scheme for the barbell

273 back squat. The second explanation could have been the higher set-volume on the muscles in the

274 legs by the "support" exercises prescribed in the protocol. The protocol prescribed both multi-

275 joint, such as lunges, and single-joint exercises, such as leg extensions, focused on the legs (Table

276 1). Some researchers argue these have to be counted in the weekly sets on the muscles (Schoenfeld

277 et al., 2019a). The chest muscles were only trained by the bench press, with three weekly sets of a

278 triceps exercise as a "support exercise."

279 Although the current findings suggest that exercise frequency does not have an overall effect by

280 itself on muscle strength, it can be an important variable to consider when developing training

281 programs. As the level of athletes increases, manipulation of training variables becomes more 
282 important (Kraemer \& Ratamess, 2004). One of the methods to ensure further adaptation for 283 athletes when the training level increases, is to correspondingly increase the total weekly volume.

284 This can be done in different ways, such as increasing weekly sets, repetitions per set, and load 285 (Kraemer \& Ratamess, 2004). When the total weekly sets for an athlete reaches an upper limit, it 286 could be advantageous to spread volume over several training sessions, as suggested by Hartmann 287 et al. (2007), to reduce the likelihood of overtraining. Exercising at too high of a volume per session 288 can be less effective at maximizing muscle adaptations. There is a limit to the number of good 289 quality sets due to fatigue (Boyas \& Guevel, 2011), but this threshold is different for each 290 individual. Some studies have shown favorable outcomes to strength when training at a lower 291 number of sets per session is introduced (Amirthalingam et al., 2017). Amirthalingam et al. (2017) 292 concluded that exercising at 4-6 sets per muscle group within a workout was optimal for muscular 293 adaptations and increasing the number of sets within a session to greater than this number did not 294 appear to produce a greater effect. An increase in total training volume (repetitions $\mathrm{x}$ set $\mathrm{x}$ 295 intensity) in one session and, therefore, nearer to failure has also been shown to significantly 296 increase the recovery time needed (Pareja-Blanco et al., 2018). In our study, a recovery time effect 297 did not occur, since the total training volume was at a medium level (Weekly sets were at 11) and 298 the intra-session sets were also low, with 5-6 sets per session for the SPLIT group and 2-3 for the 299 FULLBODY group. This effect could be the reason why the Norwegian Frequency Project showed 300 positive effects of higher training frequency (Raastad, 2012), because higher level/elite athletes 301 need a higher weekly set volume to get adaptations. However, this is speculation, because the 302 methods of that study cannot be verified or controlled. There is a possibility that frequency can 303 have an effect when weekly sets are very high, but further research is required to develop an 
304 understanding of this; as of this publication (Raastad, 2012), no studies have been conducted on

305 very high weekly sets (i.e., more than 20 sets).

306 The present findings also contradict motor learning theory, i.e., that practicing an exercise more

307 frequently will induce higher strength gains due to higher improvement in neural efficiency (Shea

308 et al., 2000). Our findings follow the hypothesis of Sale (1988) that this effect is limited for trained

309 subjects. Our results demonstrate that practicing a strength exercise twice a week could be

310 proficient to increase neural efficiency for trained subjects.

311 Fatigue could influence strength gain and recovery of athletes due to the total training volume per

312 muscle group per session. To this end, the RPE can be an important tool for resistance-trained

313 subjects and coaches during exercise execution or training sessions (Foster et al., 2001). We found

314 that subjects reported RPE changes during the training period per week in all three measurements

315 (RPE after the training bout, barbell back squat, and bench press), which is an indication that the

316 intensity changed throughout the training period, especially around week four and five. This

317 follows previous studies that have shown a correlation between the reported RPE and the intensity

318 prescribed for 1RM under resistance training (Naclerio et al., 2011; Pincivero et al., 2003). The

319 main changes in RPE per week occurred after the barbell back squat with the indication that the

320 subjects reported higher RPE after the squat for the SPLIT group then the FULLBODY group

321 (Figure 3) throughout the training period. This difference between the exercises RPE indicates that

322 a higher number of sets with barbell back squats in one session can induce higher fatigue; splitting

323 the total sets of barbell back squat into two sessions can be favorable for perceived exertion. This

324 result could also be an effect of the number of sets done with "support exercise" and single-joint

325 exercises in the training protocol. The increased number of sets done on the muscles that are used

326 can increase the subjects' fatigue and increase the recovery time needed after the training session. 
327 This study had several limitations. First, the study only lasted 8 weeks. Although the duration was

328 sufficient to achieve a significant increase in strength for both barbell back squat and bench press,

329 over a longer training time, differences between the groups could occur. Second, the small sample

330 size affected the statistical power, as most longitudinal studies in this field. Third, the results are

331 specific to resistance-trained men. Men and women could have a difference in fatigability (16). It

332 has been suggested that women have a quicker recovery rate on muscle fatigue than men after

333 resistance training (Judge \& Burke, 2010), and therefore may experience better effects of higher

334 training frequency than men. However, to our knowledge, there are no studies testing the effects

335 of training frequency on trained women. Fourth, this study did not control for the dietary intakes

336 of the subjects. The subjects may not have had an optimal nutritional intake during the training

337 period, which may affect the results; however, the randomization of the subjects should have

338 prevented such a bias.

339 Based on our findings, we conclude that training with a frequency of two and four sessions per

340 muscle group are both viable approaches to increase muscle strength in the barbell back squat and

341 bench press for trained males, as long as the total weekly training volume is equal. It is possible

342 that spreading the weekly volume to different days could be favorable for the rating of perceived

343 exertion, especially for exercising the muscles in the lower body. The group with a training

344 frequency of four reported a lower RPE for barbell back squat than the group training with a

345 frequency of two. This study corresponds with previous studies and with two meta-analyses on

346 frequency training; it seems that the effect of increasing the training frequency does not have an

347 equally important role as volume and load on strength gains (Grgic et al., 2018; Ralston et al., 348 2018). 


\section{CONCLUSION}

351 The results of this study suggest that both training with a frequency of two and four times per week

352 provides similar increases in maximal strength for trained subjects under the same total weekly

353 volume. The RPE result in this study suggests that it could be favorable to spread the total training

354 volume in several training bouts throughout the week, especially for training the muscles in the

355 lower body. This suggests that higher training frequencies could be used as a tool to counteract

356 perceived exertion for athletes since the training volume per session will be lower. Both results

357 give coaches and athletes greater variety in how to structure a training program with different

358 training frequencies without sacrificing an increase in performance. Programs can then be

359 periodized with different training frequencies to follow the athlete's personal preferences, time

360 constraints, or when the daily training volume is no longer manageable.

362

363

364

365

366

367

368

369

370

371

372

373

374

375

376

377

378

379

380

\section{REFERENCES}

American College of Sports Medicine. 2009. American College of Sports Medicine position stand. Progression models in resistance training for healthy adults. Medicine Science Sports \& Exercise 41:687-708. 10.1249/MSS.0b013e3181915670

Amirthalingam T, Mavros Y, Wilson GC, Clarke JL, Mitchell L, and Hackett DA. 2017. Effects of a Modified German Volume Training Program on Muscular Hypertrophy and Strength. Journal of Strength and Conditioning Research 31:3109-3119. 10.1519/JSC.0000000000001747

Boyas S, and Guevel A. 2011. Neuromuscular fatigue in healthy muscle: underlying factors and adaptation mechanisms. Annals of Physical and Rehabilitation Medicine 54:88-108. 10.1016/j.rehab.2011.01.001

Brigatto FA, Braz TV, Zanini T, Germano MD, Aoki MS, Schoenfeld BJ, Marchetti PH, and Lopes CR. 2019. Effect of Resistance Training Frequency on Neuromuscular Performance and Muscle Morphology After 8 Weeks in Trained Men. Journal of Strength and Condtioning Research 33:2104-2116. 10.1519/JSC.0000000000002563

Cohen J. 1988. Statistical power analysis for the behavioral sciences. Hillsdale, NJ, England: Lawrence Erlbaum Associates.

Colquhoun RJ, Gai CM, Aguilar D, Bove D, Dolan J, Vargas A, Couvillion K, Jenkins NDM, and Campbell BI. 2018. Training Volume, Not Frequency, Indicative of Maximal 
381

382

383

384

385

386

387

388

389

390

391

392

393

394

395

396

397

398

399

400

401

402

403

404

405

406

407

408

409

410

411

412

413

414

415

416

417

418

419

420

421

422

423

424

425

426

Strength Adaptations to Resistance Training. Journal of Strength and Condtioning Research 32:1207-1213. 10.1519/JSC.0000000000002414

Dankel SJ, Mattocks KT, Jessee MB, Buckner SL, Mouser JG, Counts BR, Laurentino GC, and Loenneke JP. 2017. Frequency: The Overlooked Resistance Training Variable for Inducing Muscle Hypertrophy? Sports Medicine 47:799-805. 10.1007/s40279-016-06408

Foster C, Florhaug JA, Franklin J, Gottschall L, Hrovatin LA, Parker S, Doleshal P, and Dodge C. 2001. A new approach to monitoring exercise training. Journal of Strength and Condtioning Research 15:109-115.

Gentil P, Fisher J, Steele J, Campos MH, Silva MH, Paoli A, Giessing J, and Bottaro M. 2018. Effects of equal-volume resistance training with different training frequencies in muscle size and strength in trained men. PeerJ 6:e5020. 10.7717/peerj.5020

Gomes GK, Franco CM, Nunes PRP, and Orsatti FL. 2019. High-Frequency Resistance Training Is Not More Effective Than Low-Frequency Resistance Training in Increasing Muscle Mass and Strength in Well-Trained Men. Journal of Strength and Condtioning Research 33 Suppl 1:S130-S139. 10.1519/JSC.0000000000002559

Grgic J, Schoenfeld BJ, Davies TB, Lazinica B, Krieger JW, and Pedisic Z. 2018. Effect of Resistance Training Frequency on Gains in Muscular Strength: A Systematic Review and Meta-Analysis. Sports Medicine 48:1207-1220. 10.1007/s40279-018-0872-x

Haff G, Triplett NT, and National Strength \& Conditioning Association (U.S.). 2016. Essentials of strength training and conditioning. Champaign, IL: Human Kinetics.

Hartmann M, Clarke B, Bembens D, Kilgore J, and Bemben M. 2007. Comparisons Between Twice-Daily and Once-Daily Training Sessions in Male Weight Lifters. International Journal of Sports Physiology and Performance 14:159-169.

Heaselgrave SR, Blacker J, Smeuninx B, McKendry J, and Breen L. 2019. Dose-Response Relationship of Weekly Resistance-Training Volume and Frequency on Muscular Adaptations in Trained Men. International Journal of Sports Physiology and Performance 14:360-368. 10.1123/ijspp.2018-0427

Helms ER, Cronin J, Storey A, and Zourdos MC. 2016. Application of the Repetitions in Reserve-Based Rating of Perceived Exertion Scale for Resistance Training. Strength and Conditioning Journal 38:42-49. 10.1519/SSC.0000000000000218

Judge LW, and Burke JR. 2010. The effect of recovery time on strength performance following a high-intensity bench press workout in males and females. International Journal of Sports Physiology and Performance 5:184-196. 10.1123/ijspp.5.2.184

Kraemer WJ, and Ratamess NA. 2004. Fundamentals of resistance training: progression and exercise prescription. Medicine Science Sports \& Exercise 36:674-688. 10.1249/01.mss.0000121945.36635.61

Lasevicius T, Schoenfeld BJ, Grgic J, Laurentino G, Tavares LD, and Tricoli V. 2019. Similar Muscular Adaptations in Resistance Training Performed Two Versus Three Days Per Week. Journal of Human Kinetics 68:135-143. 10.2478/hukin-2019-0062

Mclester J, Bishop E, and Guilliams M. 2000. Comparison of 1 day and 3 days per week of equal-volume resistance training in experienced subjects. Journal of Strength and Condtioning Research 14:273-281.

Morishita S, Yamaucgi S, Fujusaqa C, and Domen K. 2013. Rating of Perceived Exertion for Quantification of the Intensity of Resistance Exercise. International Journal of Physical Medicine and Rehabilitation 1:172. 
427

428

429

430

431

432

433

434

435

436

437

438

439

440

441

442

443

444

445

446

447

448

449

450

451

452

453

454

455

456

457

458

459

460

461

462

463

464

465

466

467

468

469

470

471

Naclerio F, Rodriguez-Romo G, Barriopedro-Moro MI, Jimenez A, Alvar BA, and Triplett NT. 2011. Control of resistance training intensity by the OMNI perceived exertion scale. Journal of Strength and Condtioning Research 25:1879-1888. 10.1519/JSC.0b013e3181e501e9

Pareja-Blanco F, Rodriguez-Rosell D, Aagaard P, Sanchez-Medina L, Ribas-Serna J, MoraCustodio R, Otero-Esquina C, Yanez-Garcia JM, and Gonzalez-Badillo JJ. 2018. Time Course of Recovery From Resistance Exercise With Different Set Configurations. Journal of Strength and Condtioning Research Online ahead of print. 10.1519/JSC.0000000000002756

Pincivero DM, Coelho AJ, and Campy RM. 2003. Perceived exertion and maximal quadriceps femoris muscle strength during dynamic knee extension exercise in young adult males and females. European Journal of Applied Physiology 89:150-156. 10.1007/s00421-0020768-0

Ralston GW, Kilgore L, Wyatt FB, and Baker JS. 2017. The Effect of Weekly Set Volume on Strength Gain: A Meta-Analysis. Sports Medicine 47:2585-2601. 10.1007/s40279-0170762-7

Ralston GW, Kilgore L, Wyatt FB, Buchan D, and Baker JS. 2018. Weekly Training Frequency Effects on Strength Gain: A Meta-Analysis. Sports Medicine Open 4:36. 10.1186/s40798-018-0149-9

Rhea MR, Alvar BA, Burkett LN, and Ball SD. 2003. A meta-analysis to determine the dose response for strength development. Medicine Science Sports \& Exercise 35:456-464. 10.1249/01.MSS.0000053727.63505.D4

Raastad TK, A; Wolf, D; Paulsen, G. 2012. Powerlifters improved strength and muscular adaptations to a greater extent when equal total training volume was divided into 6 compared to 3 training sessions per week (abstract). 17th annual conference of the ECSS, Brugge 4-7 July. Brugge: ECSS. p 17-2076.

Sale DG. 1988. Neural adaptation to resistance training. Medicine Science Sports \& Exercise 20:S135-145. 10.1249/00005768-198810001-00009

Saric J, Lisica D, Orlic I, Grgic J, Krieger JW, Vuk S, and Schoenfeld BJ. 2019. Resistance Training Frequencies of 3 and 6 Times Per Week Produce Similar Muscular Adaptations in Resistance-Trained Men. Journal of Strength and Condtioning Research 33 Suppl 1:S122-S129. 10.1519/JSC.0000000000002909

Schoenfeld BJ, Grgic J, Haun C, Itagaki T, and Helms ER. 2019a. Calculating Set-Volume for the Limb Muscles with the Performance of Multi-Joint Exercises: Implications for Resistance Training Prescription. Sports 7. 10.3390/sports 7070177

Schoenfeld BJ, Grgic J, and Krieger J. 2019b. How many times per week should a muscle be trained to maximize muscle hypertrophy? A systematic review and meta-analysis of studies examining the effects of resistance training frequency. Journal of Sports Science 37:1286-1295. 10.1080/02640414.2018.1555906

Schoenfeld BJ, Ratamess NA, Peterson MD, Contreras B, and Tiryaki-Sonmez G. 2015. Influence of Resistance Training Frequency on Muscular Adaptations in Well-Trained Men. Journal of Strength and Condtioning Research 29:1821-1829. 10.1519/JSC.0000000000000970

Shea C, Lai Q, Black C, and Park J. 2000. Spacing practice sessions across days benefits the learning of motor skills. Human Movement Science 19:737-760. 
472 Suchomel TJ, Nimphius S, and Stone MH. 2016. The Importance of Muscular Strength in

473

474

475

476

477

478

479

480

481

482

483

484 Athletic Performance. Sports Medicine 46:1419-1449. 10.1007/s40279-016-0486-0 Wernbom M, Augustsson J, and Thomee R. 2007. The influence of frequency, intensity, volume and mode of strength training on whole muscle cross-sectional area in humans. Sports Medicine 37:225-264. 10.2165/00007256-200737030-00004

Winett RA, and Carpinelli RN. 2001. Potential health-related benefits of resistance training. Preventive Medicine 33:503-513. 10.1006/pmed.2001.0909

Zourdos MC, Klemp A, Dolan C, Quiles JM, Schau KA, Jo E, Helms E, Esgro B, Duncan S, Garcia Merino S, and Blanco R. 2016. Novel Resistance Training-Specific Rating of Perceived Exertion Scale Measuring Repetitions in Reserve. Journal of Strength and Condtioning Research 30:267-275. 10.1519/JSC.0000000000001049 


\section{Figure legend}

\section{Figure 1.}

487 Mean 1RM of A) squat and B) bench press ( \pm SD) at pre- and posttest for each subject and the 488 average of SPLIT and FULLBODY training groups

$489 *$ indicates a significant increase in $1 \mathrm{RM}$ from pretest for this group at $\mathrm{p}<0.05$.

490

491 Figure 2.

492 Absolute individual change from pretest to posttest in A) 1RM squat and B) bench press 493 performance with average per group (dotted line).

495 Figure 3.

496 Average $( \pm \mathrm{SD})$ rating of perceived exertion for $\mathrm{A})$ whole training bout, after $\mathrm{B})$ barbell back squat, 497 and C) bench press per week.

$498 *$ indicates a significant difference between the two groups for this week at $\mathrm{p}<0.05$.

$499 \rightarrow$ indicates a significant difference from this RPE with the next one at $p<0.05$. 


\section{Table $\mathbf{1}$ (on next page)}

Table 1. Schematic overview of the training protocol.

Table 1. Schematic overview of the training protocol. 
2

3 Table 1. Schematic overview of the training protocol for each group (SPLIT and FULLBODY).

\begin{tabular}{|c|c|c|c|c|c|c|c|c|}
\hline Protocol & Day 1 & Sets & Day 2 & Sets & Day 3 & Sets & Day 4 & Sets \\
\hline \multirow[t]{6}{*}{ SPLIT } & Bench press & 6 & Back squat & 6 & Bench press & 5 & Back squat & 5 \\
\hline & Bent over row & 3 & $\begin{array}{l}\text { Stiff legged } \\
\text { deadlift }\end{array}$ & 3 & Lat pulldown & 3 & Leg press & 3 \\
\hline & $\begin{array}{l}\text { One arm } \\
\text { dumbbell row }\end{array}$ & 3 & Lunges & 3 & $\begin{array}{l}\text { Seated cable } \\
\text { row }\end{array}$ & 3 & Leg curl & 3 \\
\hline & Overhead press & 3 & $\begin{array}{l}\text { Leg } \\
\text { extension }\end{array}$ & 3 & Lateral raises & 3 & Calf raises & 3 \\
\hline & $\begin{array}{l}\text { Biceps curl } \\
\text { with dumbbells }\end{array}$ & 3 & & & $\begin{array}{l}\text { Standing cable } \\
\text { triceps curl }\end{array}$ & 3 & & \\
\hline & Face pulls & 3 & & & Face pulls & 3 & & \\
\hline \multirow[t]{6}{*}{ FULLBODY } & Back squat & 3 & Bench press & 3 & Back squat & 3 & Bench press & 3 \\
\hline & Bench press & 3 & Back squat & 3 & Bench press & 2 & Back squat & 2 \\
\hline & Bent over row & 3 & $\begin{array}{l}\text { Seated cable } \\
\text { row }\end{array}$ & 3 & $\begin{array}{l}\text { One arm } \\
\text { dumbbell row }\end{array}$ & 3 & $\begin{array}{l}\text { Lat } \\
\text { pulldown }\end{array}$ & 3 \\
\hline & Leg curl & 3 & $\begin{array}{l}\text { Leg } \\
\text { extension }\end{array}$ & 3 & Leg press & 3 & $\begin{array}{l}\text { Stiff-leg } \\
\text { deadlift }\end{array}$ & 3 \\
\hline & Biceps & 3 & Overhead & 3 & Triceps & 3 & Lunges & 3 \\
\hline & Face pulls & 3 & Calf raises & 3 & Face pulls & 3 & $\begin{array}{l}\text { Lateral } \\
\text { raises }\end{array}$ & 3 \\
\hline
\end{tabular}




\section{Table 2 (on next page)}

Table 2. Schematic overview of the load and repetitions ranges per week.

Table 2. Schematic overview of the load and repetitions ranges per week. 
1 Table 2. Schematic overview of the load and repetitions ranges per week. Both groups had a

2 predetermined program with preset weights, based on a percentage of 1RM. During each week,

3 both groups did the same total volume (sets $\mathrm{x}$ repetitions $\mathrm{x}$ intensity) in bench press and back 4 squats.

\section{Back Squat}

\section{Bench press}

\begin{tabular}{lccccc}
\hline Week & Weekly & Rep range & Intensity $(\%$ of & Rep range & Intensity $(\%$ of \\
& Sets & & 1 RM) & $1 \mathrm{RM})$ \\
\hline $\mathbf{1}$ & 11 & $5-7$ & $70-75 \%$ & $7-10$ & $65-70 \%$ \\
\hline $\mathbf{2}$ & 11 & $3-7$ & $72.5-80 \%$ & $6-10$ & $67-72.5 \%$ \\
\hline $\mathbf{3}$ & 11 & $3-6$ & $75-80 \%$ & $6-10$ & $70-75 \%$ \\
\hline $\mathbf{4}$ & 11 & $2-5$ & $77.5-87.5 \%$ & $4-8$ & $72.5-80 \%$ \\
\hline $\mathbf{5}$ & 11 & $3-5$ & $75-82.5 \%$ & $3-5$ & $72.5-82.5 \%$ \\
\hline $\mathbf{6}$ & 11 & $3-5$ & $75-85 \%$ & $3-5$ & $77.5-85 \%$ \\
\hline $\mathbf{7}$ & 11 & $2-4$ & $77.5-87.5 \%$ & $2-5$ & $77.5-87.5 \%$ \\
\hline $\mathbf{8}$ & 11 & $2-5$ & $77.5-90 \%$ & $2-4$ & $80-90 \%$
\end{tabular}

5

6

7 
Figure 1

Mean 1RM of squat and bench press ( \pm SD) at pre- and posttest for SPLIT and FULLBODY training groups

Mean 1RM of $A$ ) squat and $B$ ) bench press ( \pm SD) at pre- and posttest for each subject and the average of SPLIT and FULLBODY training groups * indicates a significant increase in 1RM from pretest for this group at $p<0.05$.

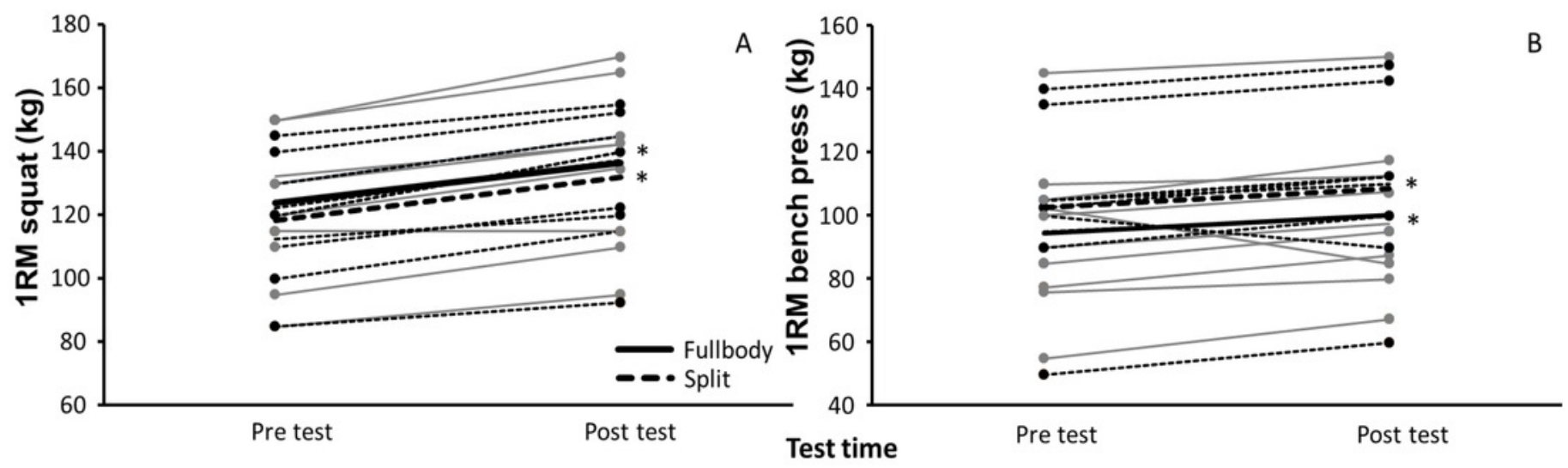




\section{Figure 2}

Absolute individual change from pretest to posttest in 1RM squat and bench press performance with average per group (dotted line).

Absolute individual change from pretest to posttest in A) 1RM squat and B) bench press performance with average per group (dotted line).
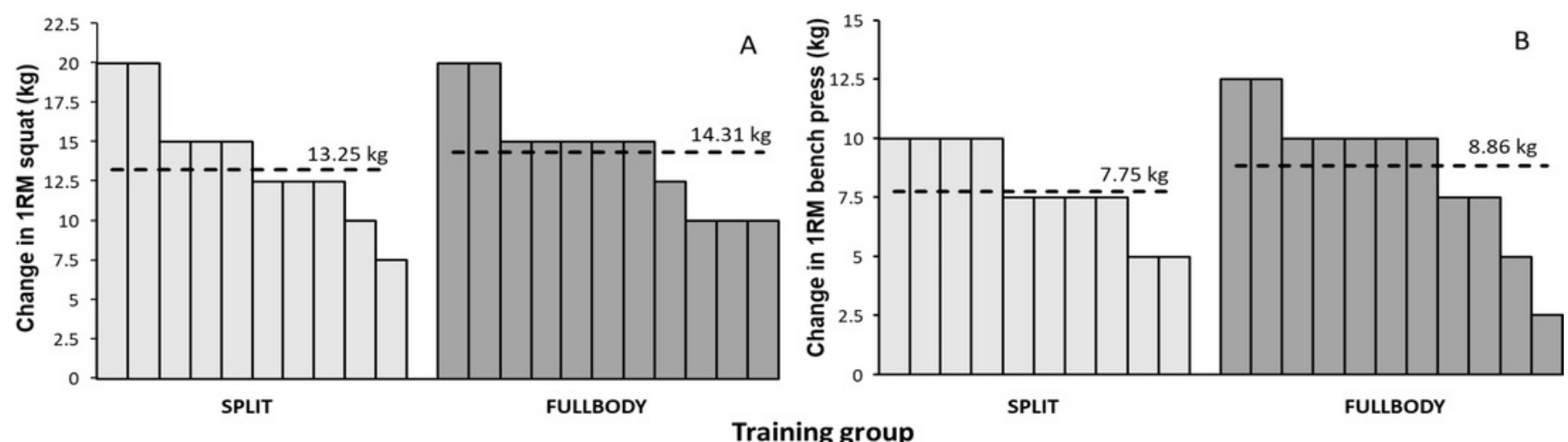


\section{Figure 3}

Average $( \pm S D)$ rating of perceived exertion for whole training bout, after barbell back squat, and bench press per week.

Average ( $\pm \mathrm{SD}$ ) rating of perceived exertion for $\mathrm{A}$ ) whole training bout, after $\mathrm{B}$ ) barbell back squat, and $\mathrm{C}$ ) bench press per week. * indicates a significant difference between the two groups for this week at $p<0.05$. $\rightarrow$ indicates a significant difference from this RPE with the next one at $p<0.05$. 

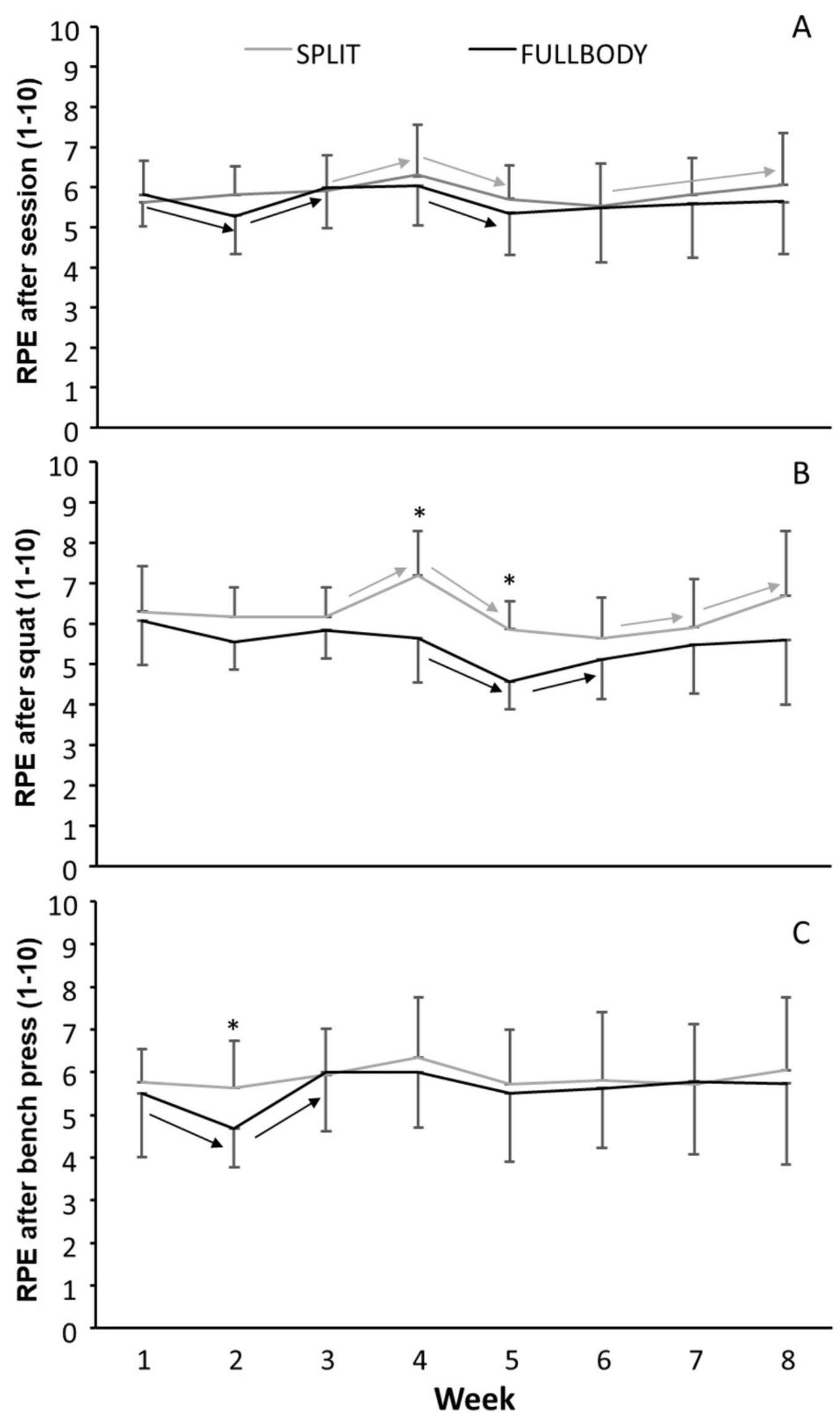